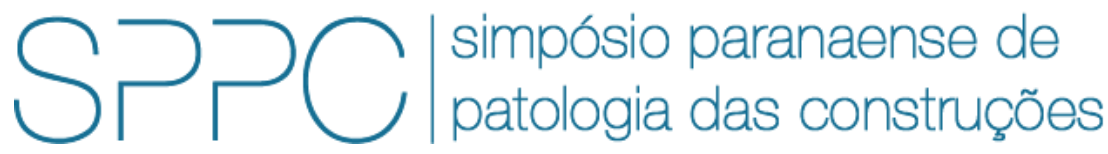

ISSN 2526-7248 artigo 2SPPC1029, pp. 354-364, 2017

\title{
Modelo matemático de ranqueamento: Avaliação do gabarito das pontes
}

\author{
Cezar Falavigna ${ }^{1}$, André Luiz Tonso Fabiani² \\ 1 Discente em Engenharia Civil, Universidade Federal do Paraná, cezarfalavigna@gmail.com \\ 2 Professor Doutor, Universidade Federal do Paraná, andre.dhs@ufpr.br
}

Resumo: O principal objetivo de uma ponte é transpor um curso água para conectar regiões por meio de uma rodovia ou ferrovia. No entanto, um dos principais causadores de colapso em obras de arte especiais (OAE's) são os efeitos gerados pelo escoamento da água do rio. Um desses efeitos é quando a altura do escoamento é superior ao gabarito (vertical e horizontal) que a estrutura possui. Isso pode ocasionar uma espécie de represamento do rio, ou, em um caso mais crítico, remover o tabuleiro e vigas dos pilares. Para evitar essa situação, um modelo de análise do gabarito deve ser desenvolvido. Esse artigo propõe uma metodologia que, inclusa no modelo de avaliação de obras de arte especiais desenvolvido no Escritório Modelo de Engenharia Civil (EMEA) da Universidade Federal do Paraná (UFPR), é possível checar se a ponte apresenta um cenário preocupante ou não.

Palavras-chave: Obras de arte especiais, Pontes, Segurança hídrica, Gabarito.

Abstract: The objective of a bridge is to transpose an obstacle, a river, to connect two different regions. Therefore, the main reason that bridges fall are due to hydraulic effects. One of those is when the river's flow rate is above what the bridge's aperture (vertical and horizontal) can drain. That can dam the river or even lift the slab and beams from the piers. To avoid this situation, an analysis model must be developed. This article proposes a methodology that suits on the bridge evaluation model developed by Federal State University (UFPR) Civil Engineering Model Office (EMEA) to check if the bridge has a worrisome scenario or not.

Keywords: Bridges, Hydraulic security, Templet. 


\section{Introdução}

O maior problema que pode ocorre com uma ponte após sua concepção, projeto, construção seria seu colapso. A falta dessa obra de arte especial (OAE) na malha rodoviária ou ferroviária pode fazer viagens ficarem mais longas, custos mais caros e até mesmo isolar uma região de receber bens e serviços.

Um estudo realizado por Wardhana e Hadipriono [1] envolvendo cerca de 500 obras que entraram em colapso nos Estados Unidos entre 1989 e 2000 notou que quase $53 \%$ dos colapsos foram por inundações e erosão. Itens como sobrecarga e impacto de embarcações, caminhões ou trens somaram $20 \%$. Chang [2] realizou um estudo envolvendo 383 falhas, dos quais $24,5 \%$ foram relacionados aos pilares e $71,8 \%$ aos encontros. As causas dos colapsos foram divididas em $38,8 \%$ para deficiências no canal, $29,6 \%$ à mudança vigorosa do escoamento, $20,0 \%$ ocasionado por detritos e somente $4,7 \%$ decorrentes de problemas estruturais.

Por essas razões, analisar a vulnerabilidade das pontes em relação a parte hidráulica deve ser desenvolvida. Porém olhar somente esse aspecto ignorando os demais seria incorrer em uma avaliação da qual se excluiria todos os campos envolvidos na área. Então, incluir a análise em um modelo que enumera holisticamente a estrutura é essencial. O modelo matemático escolhido foi o desenvolvido pelo Escritório Modelo de Engenharia Civil - EMEA da Universidade Federal do Paraná (UFPR).

\section{Metodologia}

\subsection{Modelo matemático EMEA/UFPR}

O modelo desenvolvido no EMEA/UFPR foi baseado no COST 345 [3] do CEB e já é empregado na Eslovênia e Áustria. Ele consiste em ponderar sobre os seguintes parâmetros, dano, elemento estrutural, intensidade do dano, extensão da propagação nos elementos e urgência de reparo. A formulação é descrita a seguir (Eq. 1):

$$
\sum V_{d}=\sum B_{i} * K_{1 i} * K_{2 i} * K_{3 i} * K_{4 i}
$$

Onde:

$\mathrm{Vd}=$ valor do dano;

$\mathrm{Bi}$ = Valor associado ao tipo de dano "i" sobre a segurança e/ou durabilidade;

$\mathrm{K} 1 \mathrm{i}$ = fator do elemento da estrutura, em função da sua importância no contexto da estrutura como um todo;

$K_{2 i}=$ fator indicativo da intensidade do tipo de dano " $i$ ";

$\mathrm{K} 3 \mathrm{i}$ = fator relativo à extensão da propagação do tipo de dano " $\mathrm{i}$ " nos elementos inspecionados; e

$\mathrm{K} 4 \mathrm{i}$ = fator enfatizante da urgência de intervenção para o dano " $i$ ". 
Cada um desses parâmetros possue um intervalo de valores nos quais se encaixam, descritos no "Bulletin" 243 do CEB e resumido para cada índice na tabela 1, a seguir:

Tabela 1: Intervalo de valores dos parâmetros do modelo

\begin{tabular}{cc}
\hline Parâmetro & Intervalo \\
\hline B - tipo de dano (manifestação patológica) & 1,00 a 5,00 \\
K1 - fator do elemento da estrutura & 0,10 a 0,60 \\
K2 - fator indicativo da intensidade do dano & 0,00 a 2,00 \\
K3 - fator relativo à extensão da propagação do dano & 0,50 a 2,00 \\
K4 - fator enfatizante da urgência da intervenção & 1,00 a 5,00 \\
\hline
\end{tabular}

No entanto, retirar uma nota apenas usando os valores dos índices acima não é representivo para comparações, pois as pontes possuem diferentes morfologias. Ou seja, uma ponte com mais vigas, pilares, e outros, apresenta um resultado pior que uma com menos elementos. Um caso exemplo é a comparacação de uma OAE multivigas com uma de viga caixão, a primeira apresenta no mínimo mais que duas vigas enquanto a outra apresenta um.

Para sanar esse problema a nota obtida pelos parâmetros deve ser divida por um valor de referência, assim é possível obter um índice de performance. $O$ valor de referência é atribuído a todos os danos que podem ocorrer na estrutura, multiplicados pelos valores máximos de intenside e extensão e pelo valor unitário de urgência (K4 $=1,00)$. O índice de performance é equacionado a seguir (Eq. 2):

$$
I_{p}=\frac{\sum V_{d}}{\sum V_{d, r e f}}
$$

Ip = índice de performance;

$\mathrm{Vd}=$ Valor do dano; e

Vd,ref $=$ valor de referência .

Explanado sobre o modelo de avaliação desenvolvido no EMEA/UFPR, é necessário então incrementar a análise hidráulica nesse. Esta é importante ser analisada em três partes, se o gabarito é suficiente para o escoamento do rio, se a erosão nos pilares não atinge níveis preocupantes e o objeto deste artigo que é o incremento da força horizontal nos pilares em decorrência do acúmulo de detritos.

\subsection{Análise da segurança hidráulica no modelo EMEA/UFPR}

Como citado anteriormente, a avaliação dos quesitos hidráulicos será feita em 3 partes, a análise do incremento de força horizontal nos pilares, a erosão no leito do rio gerada pelos pilares e a análise do gabartio que a ponte possui, porém nesse artigo só será relatado o incremento de força horizontal nos pilareso último item. No modelo EMEA/UFPR em qual uma das análises são relativas ao mesmo elemento da ponte, a segurança hidráulica. Isso implica que o $\mathrm{K} 1$ sempre será igual para os 3, no caso igual a 0,40. No entanto, B, K2, K3 e K4 são próprios de cada avaliação, conforme a manifestação patológica descrita. 
FALAVIGNA, C.; FABIANI, A. L. T., MODELO MATEMÁTICO DE RANQUEAMENTO: AVALIAÇÃO DO GABARITO DAS PONTES. $2^{\circ}$ Simpósio Paranaense de Patologia das Construções (20 SPPC), artigo 2SPPC1029, pp. 354-364, 2017. DOI:

Inicialmente será descrito como é feita a ponderação da análise e o embasamento teórica para se chegar a tal, em seguida, é feito a explanado como aplicar o diagnóstico no modelo matemático do EMEA/UFPR.

\subsubsection{Gabarito horizontal e vertical das pontes}

O gabarito só será importante hidraulicamente se esse não for suficiente para escoar a vazão que o rio possui. Isso ocorre se a ponte, de alguma maneira, exercer controle sobre o escoamento. Esse controle, caso exista, é feito por meio do estreitamento gerado pelos pilares. É comum na concepção do projeto estabelecer o gabarito horizontal desassociado do gabarito vertical, porém quando acontece um controle, isso se mostra errôneo. Na explicação de como aplicar no modelo EMEA/UFPR, fica mais claro como os dois estão ligados.

Primeiramente, deve-se estabelecer um valor para a manifestação patológica (B) que é analisada. Avaliando-se as manifestações que o modelo possui, o gabarito insuficiente se equipara à "Obstrução, sem causar restrições" e "Obstrução, causando restrições à movimentação". Ambas as manifestações possuem valor no modelo UFPR de 2,00, por essa razão, a manifestação gabarito não adequado é atribuído o mesmo valor.

Tabela 2: Valores de comparativos de B

\begin{tabular}{cc}
\hline Nome & B \\
\hline Obstrução, sem causar restrições & 2,00 \\
Obstrução, causando restrições à movimentação & 2,00 \\
\hline
\end{tabular}

Em seguida, deve ser atribuído o valor de $\mathrm{K} 1$, que representa o elemento que é analisado. Como dito anteriormente, para a segurança hídrica, o valor será de 0,40. Em seguida, deve ser averiguado o K2, K3 e K4, no entanto, para entrar nesse mérito é preciso estabelecer uns conceitos para a avaliação.

Os pilares das pontes causam o estreitamento da seção de escoamento da água, esse estreitamento pode gerar um controle no canal do curso d'água. Para verificar se estão gerando esse controle ou não é preciso utilizar dois conceitos da hidráulica, a análise da energia que o canal possui e a altura crítica de escoamento.

Segundo Neidert et al. [5], considerando uma determinada seção em um canal, sua energia possui 3 parcelas, a cota de fundo (segundo um plano horizontal de referência), a altura de pressão $(p / y)$ correspondente ao ponto coincidente com 0 fundo do canal e a altura de velocidade $\left(\alpha^{*} U^{2} / 2 g\right)$ que é referente à energia cinética do escoamento, conforme ilustra a figura 1. 


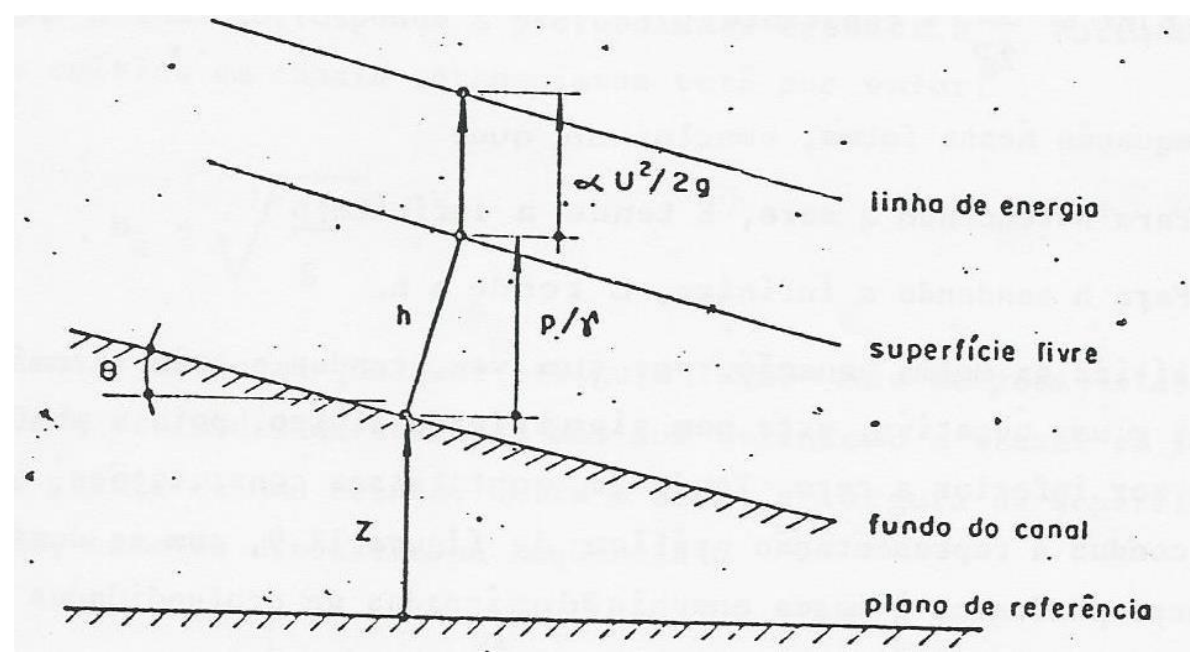

Figura 1: Energia total do canal. [5]

Se for utilizado como referência o plano de fundo do canal ao invés de um plano horizontal, pode-se eliminar a energia potencial, resultando a energia específica de um canal, que é o somatório da altura de pressão com a energia cinética. As equações 3 e 4 descrevem esse processo.

Pela soma das 3 parcelas (Figura 1) é possível saber a energia total (Eq. 3):

$$
\mathrm{E}=\mathrm{Z}+\frac{\mathrm{p}}{\gamma}+\frac{\alpha \mathrm{U}^{2}}{2 \mathrm{~g}}
$$

Onde:

$E=$ energia do canal;

$Z$ = cota de fundo;

$\mathrm{p}=$ pressão hidrostática;

$Y=$ preso específico da água;

$\alpha=$ coeficiente corretor da energia cinética;

$\mathrm{U}=$ velocidade média do escoamento; e

$\mathrm{G}=$ aceleração da gravidade.

Ao invés de ser usado um plano de referência, é possível usar o fundo do canal como tal e assim se obter a energia específica, conforme a equação 4:

$$
\mathrm{E}=\mathrm{h} \cdot \cos \theta+\alpha \frac{\mathrm{q}^{2} / \mathrm{h}^{2}}{2 \mathrm{~g}}(4)
$$

Onde: 
$\mathrm{h}$ = altura de água do escoamento;

$\theta=$ ângulo entre o plano horizontal e o fundo do canal; e

q = vazão específica do canal.

Adotando-se $\alpha=1,0$ e com a declividade suficientemente pequena $\left(\theta \approx 0^{\circ}\right)$, portanto, $\cos 0^{\circ}=1,0$, tem-se da energia específica do canal (Eq. 5):

$$
\mathrm{E}=\mathrm{h}+\frac{\mathrm{q}^{2} / \mathrm{h}^{2}}{2 \mathrm{~g}}
$$

Se a expressão ficar em função da vazão, a energia é calculada pela seguinte formulação (Eq. 6):

$$
\mathrm{E}=\mathrm{h}+\frac{\mathrm{Q}^{2} / \mathrm{A}^{2}}{2 \mathrm{~g}}
$$

Onde:

$E=$ energia do canal;

$\mathrm{h}=$ altura (profundidade) do escoamento [m];

$\mathrm{Q}=$ vazão do rio $\left[\mathrm{m}^{3} / \mathrm{s}\right]$, função de $\mathrm{h}[\mathrm{m}]$;

$A$ = área do canal, função de $\mathrm{h}$ [m]; e

$\mathrm{g}$ = aceleração da gravidade $\left[\mathrm{m}^{2} / \mathrm{s}\right]$;

Para medir essa altura, recomenda-se escolher uma seção a jusante da ponte em uma área na qual já não se perceba os efeitos hidráulicos dos pilares, ou seja, imune às perturbações na superfície d'água, normalmente 2 a 3 vezes a largura do tabuleiro.

Analisando a solução analítica da equação 5, percebe-se que esta conduz a 3 raízes, duas positivas e uma negativa, esta sem significado físico, já que a profundidade não pode ser negativa.

Com a profundidade definida, pode-se se estabelecer o número de Froude para o escoamento, utilizando o seguinte equacionamento (Eq. 7):

$$
\mathrm{F}_{\mathrm{r}}{ }^{2}=\frac{\alpha \cdot \mathrm{Q}^{2} \cdot \mathrm{B}}{\mathrm{g} \cdot \mathrm{A}^{3}}(7)
$$

Onde:

$\mathrm{Fr}=$ número de Froude;

$\alpha=$ coeficiente corretor da energia cinética; 
$\mathrm{Q}=$ vazão;

$\mathrm{B}=$ largura do canal;

$g$ = aceleração da gravidade; e

$A$ = área da seção do rio.

Utilizando o a e Fr igual a um (1,00), calcula-se o valor de h, a seguinte expressão é decorrente dessa adoção (Eq. 8):

$$
\frac{A_{(h)}^{3}}{B_{(h)}}=\frac{Q^{2}}{g}
$$

O número de Froude estabelece o limite entre o canal ser considerado fluvial ou torrencial, ou seja, se o for menor que $1(\mathrm{Fr}<1)$, o regime é fluvial (pode-se encontrar bibliografias utilizando o termo subcrítico), caso o valor seja maior que 1 (Fr $>1$ ), o regime é torrencial (supercrítico). Portanto, quando Froude for igual a 1 temos a altura crítica do escoamento. Em um canal retangular, é possível plotar um gráfico da relação entre da altura $(h)$ com a energia do canal $(E)$, assim fica mais fácil compreender a o conceito de torrencial e de fluvial, conforme ilustra a figura 2.

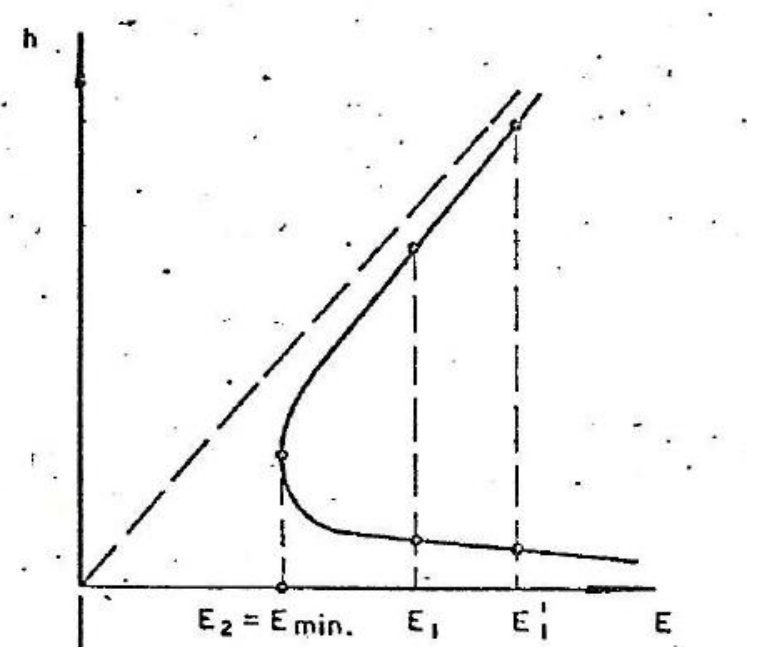

Figura 2: Relação da altura (h) com a energia (E).

No ponto E2, onde a energia é igual a mínima, o número de Froude é igual a $1(\mathrm{Fr}=$ 1), isso significa que na curva, qualquer ponto acima do desse representa um regime fluvial (subcrítico, $\mathrm{Fr}<1$ ) e qualquer ponte da curva abaixo de E2, o regime será torrencial (supercrítico, $\mathrm{Fr}>1$ ).

$\mathrm{Na}$ equação 8, como a área e a largura do canal são funções da profundidade do escoamento e com a vazão obtida em campo ou via dados da Agência Nacional de Águas (ANA), pode-se achar a altura crítica $(\mathrm{Fr}=1)$. Desta, é possível calcular a energia mínima que o escoamento possui, utilizando a fórmula de energia (Eq. 6) descrita anteriormente. 
Com o valor da energia mínima e a energia do canal (obtida com os dados de escoamento a jusante da ponte), se a energia que o rio possuir for maior que a energia mínima (Erio > Emin) então o valor de K2 será igual a zero $(\mathrm{K} 2=0)$, pois nessa situação o estreitamento ocasionado pelos pilares não gera controle sobre o escoamento. Isso faz com que a análise seja a simples relação de altura do escoamento com relação ao gabarito vertical que a estrutura possui.

Porém, caso a energia seja menor que a mínima então os pilares funcionam como controle do escoamento, a partir disso, deve-se recalcular o h1 (altura a montante) utilizando a Emín.

Essa altura é calculada utilizando a seção a montante da obra e será usada para a adoção de K2. O percentual para o K2 será decorrente da seguinte formulação (Eq. 9):

$$
\% \mathrm{~K}_{2}=\frac{\mathrm{h}_{1}-\mathrm{h}_{\text {nat }}}{\mathrm{h}_{\text {nat }}}
$$

Onde:

hnat = altura natural (medida a jusante do rio) sem influência da ponte;

h1 = altura a montante da estrutura (calculado via energia mínima).

O percentual gerado é utilizado para determinação do valor de K2 no modelo UFPR, conforme ilustrado pela tabela 2 .

Tabela 2: Intervalo de valores para o K2 - Intensidade do dano

\begin{tabular}{|c|c|c|c|}
\hline Tipo & Grau & Critério & K2 \\
\hline 0 & Insignificante & Dano irrelevante & 0,00 \\
\hline I & Pequeno & $\begin{array}{c}\text { Dano pequeno, em menos de } 10 \% \text { da área } \\
\text { total do elemento }\end{array}$ & 0,50 \\
\hline II & Médio & $\begin{array}{c}\text { Dano intermediário, confinado em uma área } \\
\text { pequena de } 10 \% \text { a } 25 \% \text { da área total do } \\
\text { elemento }\end{array}$ & 1,00 \\
\hline III & Grande & $\begin{array}{c}\text { Dano grande, de } 25 \% \text { a } 75 \% \text { da área total do } \\
\text { elemento, ou em muitos lugares de um } \\
\text { elemento estrutural }\end{array}$ & 1,50 \\
\hline IV & Imenso & $\begin{array}{c}\text { Dano muito grande, na maior parte da área de } \\
\text { um elemento, mais de } 75 \% \text { da área total do } \\
\text { elemento }\end{array}$ & 2,00 \\
\hline
\end{tabular}

Em seguida é necessário estabelecer-se o valor para a extensão de propagação, K3. A amplitude é calculada via relação entre o número de vãos na água e o número total de vãos, ou seja, quanto da estrutura está submetida aos efeitos do controle (Eq. 10). Desse percentual obtém-se o K3 conforme a tabela: 
FALAVIGNA, C.; FABIANI, A. L. T., MODELO MATEMÁTICO DE RANQUEAMENTO: AVALIAÇÃO DO GABARITO DAS PONTES. $2^{\circ}$ Simpósio Paranaense de Patologia das Construções (20 SPPC), artigo 2SPPC1029, pp. 354-364, 2017. DOI:

$$
\% K_{3}=\frac{N u ́ m e r o \text { de vãos sobre a água }}{\text { Número total de vãos na ponte }}
$$

Tabela 3: Intervalo de valores para o parâmetro K3 - extensão de propagação

\begin{tabular}{cc}
\hline Critério & K3 \\
\hline Dano aparece em menos de 10\% dos elementos estruturais da OAE & 0,50 \\
Dano aparece ente $10 \%$ e $25 \%$ dos elementos estruturais da OAE & 1,00 \\
Dano aparece entre $25 \%$ e $75 \%$ dos elementos estruturais da OAE & 1,50 \\
Dano aparece entre $75 \%$ e $100 \%$ dos elementos estruturais da OAE & 2,00 \\
\hline
\end{tabular}

Para finalizar, os valores associados à urgência da intervenção são apresentados a seguir. O dano é classificado de acordo com a tabela NúMERO a partir da avaliação do inspetor e das condições existentes em campo. Como fatores importantes de análise das condições existentes, recomenda-se ao inspetor observar se a estrutura é isostática ou hiperestática, o comprimento e número de vãos e também a relação entre o gabarito vertical e a altura obtida para h1. A partir disso pode-se atribuir a urgência de reparo conforme a tabela a seguir:

Tabela 4: Intervalo de valores para o parâmetro K4 - urgência de intervenção

\begin{tabular}{|c|c|c|}
\hline Classificação & Critério & K4 \\
\hline Não urgente & $\begin{array}{c}\text { Intervenção não urgente pois o dano não interfere na } \\
\text { utilização e capacidades da OAE, também não altera a } \\
\text { sua durabilidade }\end{array}$ & 1,00 \\
\hline Dano à reparar & $\begin{array}{c}\text { Dano deve ser reparado em período não maior que } 5 \\
\text { anos, para garantir a servicibilidade e não } \\
\text { compremeter a durabilidade }\end{array}$ & 2,50 \\
\hline Reparo imediato & $\begin{array}{c}\text { Reparo imediato, pois o dano já está comprometendo } \\
\text { a utilização da OAE, com risco às pessoas }\end{array}$ & 4,00 \\
\hline Limitação de carga & $\begin{array}{l}\text { Limitação de carga, interrupção de tráfego e } \\
\text { escoramento imediato deve ser feito, além do reparo }\end{array}$ & 5,00 \\
\hline
\end{tabular}

No último item da tabela anterior (Tabela 4), "Limitação de carga", a descrição referese ao escoramento imediato, porém para o caso da análise do gabarito, o mesmo não deve ser feito, visto que tal atitude restringiria mais o canal, intensificando o efeito do estreitamento.

Com todos os parâmetros definidos, é possível gerar valor do dano para checar se o gabarito da ponte é suficiente para o escoamento ao qual ele está imposto, e assim incluir ao modelo EMEA/UFPR.

\section{Conclusões}

O modelo desenvolvido no Escritório Modelo de Engenharia Civil - EMEA da UFPR aborda globalmente vários aspectos da estrutura, incluindo sua operação. Entretanto, como outros estudos mostram, falhas relacionadas ao corpo hídrico que as pontes transpõem são o principal causador desses colapsos. 
Por isso, é essencial a análise hidráulica da ponte, que envolve seu o gabarito disponível para passar da água. O primeiro erro é desvincular o gabarito vertical do horizontal, pois como explicitado no artigo, o estreitamento da seção incorre em um aumento do nível a montante, caso exista um controle.

Existindo essa situação, é necessário então aplicar um modelo para quantificar quão prejudicial é esse dano. No caso foi escolhido o modelo desenvolvido no EMEA/UFPR, já que abarca uma avaliação holística (inclui além da estrutura, itens como operação) e possui parâmetros estabelecidos que facilitam o enquadramento da análise nesses índices. Porém, ressalta-se que é de suma importância a adição desse aspecto no modelo.

Outro fator, é que com o gabarito da ponte estabelecido, é possível associar equações para vazão em relação a intensidade de chuva, como, por exemplo, o método racional, no qual é possível prever a partir de qual intensidade ou utilizando o hidrograma unitário, se os níveis de água são algum risco para a ponte.

Também é lembrado que para julgar de maneira mais completa, além do gabarito, existem o acréscimo de esforços horizontais nos pilares e a erosão nos pilares. Somente com esses três é possível dar uma avaliação mais acurada sobre a segurança da ponte em relação à parte hídrica.

Outro ponto que deve ser destacado, é que o modelo também deve ser avaliado em campo para checar sua viabilidade como método de inspeção, pois nem sempre é fácil ter acesso ao rio, ou mesmo, retirar as medidas para as formulações e consequentemente os parâmetros $\mathrm{K} 2, \mathrm{~K} 3$, K4.

\section{Agradecimentos}

À Universidade Federal do Paraná, ao Setor de Tecnologia da UFPR, ao Departamento de Construção Civil (DCC) - UFPR, ao Departamento de Hidráulica e Saneamento (DHS) - UFPR, ao Programa de Pós-Graduação em Engenharia de Construção Civil (PPGECC), ao Programa de Pós-Graduação em Engenharia de Recursos Hídricos (PPGERHA), o Escritório Modelo de Engenharia Civil - EMEA da UFPR e principalmente ao professor PhD. Mauro Lacerda Santos Filho por propiciar as condições necessárias para que essa pesquisa fosse realizada.

\section{Referências}

[1] WARDAHANA. Kumalasari; HADRIPRIONO, Fabian C. (2003). Analysis of recent bridge failures in the United States. Journal of Performance Of Constructed Facilities. 17:3 p. 144-150.

[2] CHANG, F.F.M. (1973). A statistical summary of the cause and cost of bridge failures. Federal Highway Administration - FHWA emergency relief files.

[3] EUROPEAN CO-OPERATION IN THE FIELD OF SCIENTIFIC AND TECHNICAL RESEARCH. (2007). COST 345: Methods used in the european states and assess the condition of highway structures. .Disponível em: 
FALAVIGNA, C.; FABIANI, A. L. T., MODELO MATEMÁTICO DE RANQUEAMENTO: AVALIAÇÃO DO GABARITO DAS PONTES. $2^{\circ}$ Simpósio Paranaense de Patologia das Construções (20 SPPC), artigo 2SPPC1029, pp. 354-364, 2017. DOI:

<http://cost345.zag.si/Reports/COST_345_WG23.pdf>. Acesso em 21 ago. 2016

[4] COMITÉ EURO-INTERNACIONAL DU BETÓN. (1998). Bulletin 243: Strategies for Testing and Assessment of Concrete Structures. Suiça

[5] NEIBEIRT, S.N., TOZZI, M.J., OTA, J.J., BOBKO, G. (1990). Noções básicas de mecânica dos fluidos e hidráulica. 2. v. Universidade Federal do Paraná, Curitiba, Brasil. 